\title{
Adverse effects of androgen deprivation therapy in men with prostate cancer: a focus on metabolic and cardiovascular complications
}

\author{
Lauren Collins and Shehzad Basaria
}

Prostate cancer ( $\mathrm{PCa}$ ) is the most common malignancy in men. Prostate being an androgen responsive tissue, androgen deprivation therapy (ADT) is used in the management of locally advanced (improves survival) and metastatic (improves pain and quality of life) PCa. Over the past two decades, the use of ADT has significantly increased as it is also being used in patients with localized disease and those experiencing biochemical recurrences, though without any evidence of survival advantage. Hypogonadism resulting from ADT is associated with decreased muscle mass and strength, increased fat mass, sexual dysfunction, vasomotor symptoms, decreased quality of life, anemia and bone loss. Insulin resistance, diabetes and cardiovascular disease have recently been added to the list of these complications. As the majority of men with PCa die of conditions other than their primary malignancy, recognition and management of these adverse effects is paramount. Here we review data evaluating metabolic and cardiovascular complications of ADT. Asian Journal of Andrology (2012) 14, 222-225; doi:10.1038/aja.2011.109; published online 20 February 2012

Keywords: androgen deprivation therapy; cardiovascular disease; diabetes; hypogonadism; prostate cancer

\section{INTRODUCTION}

Prostate cancer (PCa) is the most common cancer diagnosed in men in the United States and the second most common cause of cancer related death. Estimates suggest that approximately 240890 new cases of PCa were diagnosed in the United States in 2011 resulting in deaths of 33 720 men. ${ }^{1}$ In men with localized PCa, treatment options include watchful waiting, radical prostatectomy or radiation therapy. In patients with locally advanced disease, androgen deprivation therapy (ADT) in combination with external beam radiation or as an adjuvant therapy (post-prostatectomy and pelvic lymphadenectomy), have been shown to improve survival., ${ }^{2,3}$ In patients with distant metastases, ADT improves quality of life. ${ }^{4,5}$ Although ADT is beneficial in these patient populations, it is often used as primary therapy in men with localized disease and in men encountering biochemical recurrence ${ }^{6}$ without evidence of survival advantage. The overall use of ADT has increased in the past two decades ${ }^{7}$ and its use in men with localized disease and biochemical recurrence accounts a great deal for this increase. Since most men with PCa die of conditions other than their primary malignancy, recognition and management of these adverse effects is paramount.

\section{ADT: BODY COMPOSITION CHANGES}

Patients undergoing ADT experience increased fat mass, decreased muscle mass and decreased muscle strength. ${ }^{8,9}$ These observations are not surprising as androgens stimulate mesenchymal pluripotent stem cells towards myogenic lineage instead of adipogenic lineage ${ }^{10} \mathrm{~A}$ decade ago, a prospective study from the United Kingdom showed that ADT in 22 newly diagnosed men with PCa resulted in an increase in fat mass from $20.2 \pm 9.4 \mathrm{~kg}$ at baseline to $21.9 \pm 9.6 \mathrm{~kg}$ at 3 months $(P=0.008)$, while lean body mass decreased from $63.2 \pm 6.8 \mathrm{~kg}$ at baseline to $61.5 \pm 6.0 \mathrm{~kg}(P=0.016){ }^{11}$ These results were confirmed by another 2-year prospective study that showed an increase in total fat mass by $9.4 \%$ and reduction in lean body mass by $2.7 \% .^{9}$ A recent study showed that while changes in body composition continue throughout the course of ADT, the bulk of these changes occur during the first year of treatment. ${ }^{12}$ These investigators examined body composition in 81 men with PCa not undergoing ADT, 43 men on shortterm $\operatorname{ADT}$ ( $<6$ months), 67 men on long-term ADT ( $>6$ months) and 53 healthy aged matched controls. While the greatest change in body composition in men on ADT occurred during the first year, significant changes continued to occur even after 2 years. Men with PCa not on $\mathrm{ADT}$ and healthy age matched controls did not experience significant changes in body composition.

\section{ADT: INSULIN RESISTANCE AND DIABETES}

Weight gain is a risk factor for insulin resistance, diabetes and metabolic syndrome. ${ }^{13}$ One of the early metabolic changes related to ADT include hyperinsulinemia that is associated with an increase in fat mass. This was first shown a decade ago in 22 patients with newly diagnosed PCa. ${ }^{11}$ Fasting serum insulin increased from $11.8 \mathrm{mU}^{-1}$ at baseline to $19.3 \mathrm{mU} \mathrm{l}^{-1}$ at 3 months; however, there was no change in fasting glucose levels. Another 12 -week prospective study of 25 non-diabetic men showed a decrease in insulin sensitivity index by $12.9 \% \pm 7.9 \%$ and an increase in fasting insulin levels by $25.9 \% \pm 9.3 \%$ 
on ADT. ${ }^{14}$ A study of 49 men with PCa undergoing ADT for 6 months observed a mild, though statistically significant, increase in fasting blood glucose (from $103 \mathrm{mg} \mathrm{dl}^{-1}$ to $106 \mathrm{mg} \mathrm{dl}^{-1}$ ), although the levels remained below the diabetes range. ${ }^{15}$ This suggests that short-term $\mathrm{ADT}$ results in insulin resistance; however, this compensatory hyperinsulinemia maintains euglycemia. However, then it was unclear whether long-term ADT will eventually result in diabetes. To answer this question, a cross-sectional study recruited 18 men undergoing ADT for at least 12 months, 17 age-matched men with non-metastatic PCa who were not androgen-deprived and had undergone prostatectomy and/or radiation therapy in the past and 18 age-matched eugonadal controls without PCa. ${ }^{16}$ None of the subjects had known history of diabetes mellitus. The investigators found that in addition to hyperinsulinemia, fasting hyperglycemia was also more prevalent in the ADT group compared to the two control groups (Figure 1), with $44 \%$ of ADT patients having glucose levels in the diabetic range. The duration of ADT was directly related to the severity of these metabolic abnormalities. ${ }^{17}$ This study demonstrated that fasting hyperglycemia (including undiagnosed diabetes) is more prevalent in men undergoing ADT. A subsequent population-based study showed that men undergoing ADT are also at a higher risk of developing incident diabetes. ${ }^{18}$ This study looked at health data on 73196 men $\geqslant 66$ years old and found that men undergoing ADT experienced a $44 \%$ increase in incident diabetes compared to men not undergoing ADT after adjusting for confounders. These findings were confirmed by an age-matched cohort study using linked administrative data at the Institute for Clinical Evaluative Sciences in Ontario, Canada. ${ }^{19}$ Men were followed for an average of 6.47 years and were found to have an increased risk of diabetes compared with men who were ADT-naive. Another observational study of 37443 men with loco-regional PCa also showed that treatment with gonadotropin-releasing hormone $(\mathrm{GnRH})$ agonists was associated with a significant increase in incident diabetes (159.4 events per 1000 person-years vs. 87.5 events for no ADT).$^{20}$ Based on these findings, prospective long-term studies are needed that are specifically designed to evaluate for incident diabetes.

\section{ADT: METABOLIC SYNDROME}

Body composition changes in men undergoing ADT are probably the precursor of metabolic complications. According to the Adult Treatment Panel-III, metabolic syndrome in men is diagnosed if they have three of the following five criteria: fasting plasma glucose level of $>110 \mathrm{mg} \mathrm{dl}^{-1}$, serum triglyceride level of $\geqslant 150 \mathrm{mg} \mathrm{dl}^{-1}$, serum high density lipoprotein level of $<40 \mathrm{mg} \mathrm{dl}^{-1}$, blood pressure of $\geqslant 130 / 85 \mathrm{mmHg}$ and waist circumference $>102 \mathrm{~cm}$. The recent guidelines from the American Heart Association maintain most of these criteria; however, the cutoff for fasting glucose level has been lowered to $>100 \mathrm{mg} \mathrm{dl}^{-1} .{ }^{21}$ Patients on antihypertensive and lipid-lowering medications are also classified as positive for their respective criterion. Men with metabolic syndrome are three times more likely to die of coronary heart disease and other cardiovascular (CV) complications than their healthy counterparts. ${ }^{22}$ Recent epidemiologic studies have

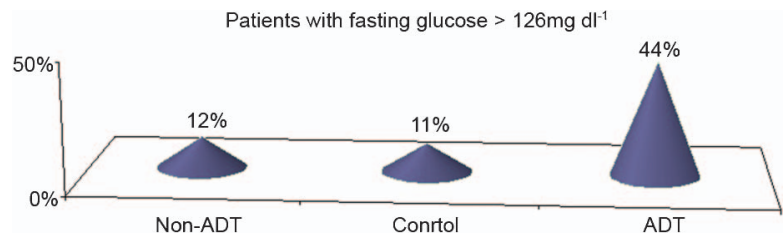

Figure 1 Prevalence of undiagnosed diabetes in men undergoing long-term androgen deprivation therapy. ${ }^{16}$ ADT, androgen deprivation therapy. shown that male hypogonadism has emerged as an independent risk factor for metabolic syndrome. ${ }^{23}$ This may also be the case in men undergoing ADT as a cross-sectional study has reported a higher prevalence of metabolic syndrome in these men. ${ }^{24}$ This study showed that metabolic syndrome was prevalent in over $50 \%$ of men undergoing long-term ADT when compared to age-matched men with $\mathrm{PCa}$ not undergoing ADT (22\%) and their age-matched eugonadal counterparts (20\%) (Figure 2). These metabolic changes could contribute to $\mathrm{CV}$ disease in men undergoing ADT.

\section{ADT: CARDIOVASCULAR DISEASE (CVD) AND MORTALITY}

The relationship between androgens and CVD remains unresolved. Findings from some epidemiological studies suggest that low serum testosterone level is associated with higher CV and all-cause mortality, ${ }^{25}$ although one cannot exclude reverse causality. Small cross-sectional studies have shown an inverse association between serum testosterone and the degree of coronary atherosclerosis; ${ }^{26}$ however, large prospective studies have yet to confirm this relationship. Recently, data have emerged implicating ADT with CVD and mortality. However; it is important to note that none of these studies was designed with $\mathrm{CV}$ disease or mortality as the primary outcome and the available data are a result of secondary analyses of large oncological trials. Here we briefly summarize these data.

In 2006, a study evaluating data from the Surveillance, Epidemiology and End Results and Medicare databases showed an association between ADT and CVD. ${ }^{18}$ The study examined records of 73 196 people $\geqslant 66$ years old diagnosed with localized PCa who were being treated with GnRH agonist or orchiectomy. The analysis showed that $\mathrm{GnRH}$ agonist therapy was associated with a significant increase in risk of incident coronary heart disease (adjusted hazard ratio: 1.16), myocardial infarction (hazard ratio: 1.11) and sudden cardiac death or life-threatening ventricular arrhythmia (hazard ratio: 1.16), while orchiectomy was associated only with a greater risk of diabetes. This could either be due to fewer numbers of patients who underwent orchiectomy or $\mathrm{GnRH}$ agonists may have a direct role in these events. The latter argument remains unresolved as one previous study showed QTc prolongation in patients receiving GnRH agonists; ${ }^{27}$ however; a recent 12-month long comparative study (GnRH agonist vs. antagonist) did not show any differences in CV events between the two groups. ${ }^{28}$ A study using the Cancer of the Prostate Strategic Urologic Research Endeavor database also showed that men receiving ADT were 2.6 times more likely to die of $\mathrm{CV}$ events than non-ADT controls. $^{29}$ Overall CVD morbidity from ADT was examined by another study using the Surveillance, Epidemiology and End Results registry that evaluated 22816 men with newly diagnosed PCa who had not experienced a CV event during the first 12 months post-PCa diagnosis. ${ }^{30}$ The study found that men who received ADT had a $20 \%$ higher risk of serious CV morbidity than similar men who did not. Another observational study of 37443 veterans (mean age: 66.9 years) with PCa showed that men undergoing ADT had higher rates of incident coronary heart disease, myocardial infarction, sudden

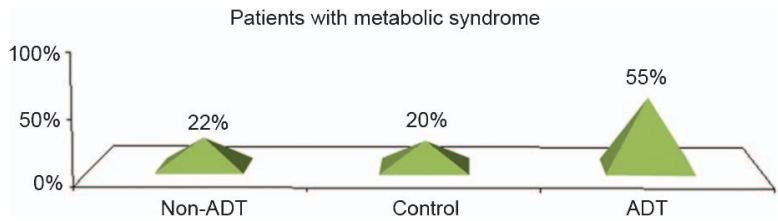

Figure 2 Prevalence of metabolic syndrome in men undergoing long-term androgen deprivation therapy. ${ }^{24}$ ADT, androgen deprivation therapy. 
death and stroke compared to men not treated with ADT. ${ }^{20}$ The progression of disease leading to potentially negative outcomes is further documented in another study of 5077 men (median age: 69.5 years) with localized or locally advanced $\mathrm{PCa} .{ }^{31}$ Men treated with neoadjuvant $\mathrm{ADT}$ who had a history of coronary artery disease-induced congestive heart failure were found to be at an increased risk for all cause mortality.

Because the same metabolic changes that predispose to coronary disease may also increase the risk of cerebrovascular events, one would expect a higher incidence of transient ischemic attacks or strokes in ADT patients. A recent population-based cohort study of 22310 patients with PCa showed that ADT may increase risk of transient ischemic attacks and stroke. ${ }^{32}$ Increased frequency of thromboembolic events have also been reported in androgen-deprived men. ${ }^{33}$

\section{ADT AND CVD: CONFLICTING DATA}

Although some studies do illustrate a relationship between ADT and increased risk of CV events, other studies have not confirmed this association. Alibhai et al. ${ }^{19}$ took a cohort of men aged 66 years or older who had been observed for a mean of 6.47 years. While ADT was associated with increased risk of diabetes, there was no such association with incidence of acute myocardial infarction in this group of nearly $20000 \mathrm{ADT}$ users matched to the same number of non-ADT users for pair analysis. ${ }^{19}$ Similar results were reported in an analysis from the Radiation Therapy Oncology Group, a large prospective randomized controlled trial of men with locally-advanced PCa comparing long-term (28 months) and short-term (4 months) ADT in combination with radiation therapy. ${ }^{34}$ The study found that longterm ADT does not increase CV mortality in men with locally advanced PCa. They did find that traditional CV risk factors suck as old age, prevalent CVD and diabetes were significantly associated with $\mathrm{CV}$ mortality. A recent large study assessing the impact of ADT on CVD mortality in men undergoing curative intent external beam radiation therapy confirmed these findings. ${ }^{35}$ Among these men, the cumulative CV mortality at 7 years was $2.6 \%$ for men not undergoing $\mathrm{ADT}, 2.1 \%$ for men with $<6$ months $\mathrm{ADT}$ and $1.4 \%$ for men undergoing ADT for $>6$ months. Punnen et al. ${ }^{36}$ analyzed a total of 7248 men in the Cancer of the Prostate Strategic Urologic Research Endeavor registry to assess the relationship between ADT and CV mortality. Competing hazard survival analysis was performed for PCa-specific, CV and all-cause mortality. Based on propensitymatching algorithm, there was no significant difference in CV mortality among men who did versus who did not receive ADT. ${ }^{36}$

A few studies have also contradicted the association of ADT with cerebrovascular disease. The first study found that men on ADT were less likely to experience a stroke than men not undergoing ADT. ${ }^{19}$ Chung et al. ${ }^{37}$ recently conducted a 5-year follow-up study in men undergoing ADT. The incidence of stroke in men who received ADT was $17.2 \%$ compared to $18.9 \%$ in men who did not. After adjusting for confounders, there was no significant difference in the hazard of stroke between the two groups.

\section{CONCLUSION}

As evident from the conflicting data, the relationship between ADT and CVD remains unclear. Until clarity is reached on this issue, it would be prudent for physicians initiating ADT to carefully weigh risk/benefit ratio. It is essential for clinicians and patients alike to be informed regarding the potential benefits and harms of ADT. Particular attention should be given to older men who have pre-existing CV comorbidities. Patients should be encouraged to adopt a healthy lifestyle such as a balanced diet and engagement in physical activity. While planning physical activity, a practitioner should consider prevalent cardiac risk in that patient. Clinicians should use evidence-based guidelines published by the American Heart Association, as suggested by a recent science advisory. ${ }^{38}$ Prospective studies evaluating the benefit of aggressive baseline CV screening, lifestyle modifications and pharmacological preventive measures (insulinsensitizing agents, lipid-lowering therapy, aspirin) in patients with PCa prior to undergoing ADT are needed. A recent pilot study of men about to receive ADT randomized them to ADT alone or 6 months of metformin and exercise program. ${ }^{39}$ After 6 months, significant improvements in abdominal girth, weight and body mass index were seen in the intervention arm.

There remains a great need to conduct prospective studies to assess $\mathrm{CV}$ risk associated with ADT. These studies should be powered for incident $\mathrm{CV}$ events and CV mortality as the primary outcome. As more light is shed on the CV risks of ADT, clinical care of these patients will undoubtedly improve.

\section{COMPETING FINANCIAL INTERESTS}

All authors declare that there are no competing financial interests.

1 Siegel R, Ward E, Brawley O, Jemal A. Cancer statistics 2011. CA Cancer J Clin 2011; 61: 212-36.

2 Bolla M, Gonzalez D, Warde P, Dubois JB, Mirimanoff RO et al. Improved survival in patients with locally advanced prostate cancer treated with radiotherapy and goserelin. N Engl J Med 1997; 337: 295-300.

3 Messing EM, Manola J, Sarodsy M, Dubois JB, Mirimanoff RO et al. Immediate hormonal therapy compared with observation after radical prostatectomy and pelvic lymphadenectomy in men with node positive prostate cancer. N Engl J Med 1999; 341: 1781-8

4 Shahani S, Braga-Basaria M, Basaria S. Androgen deprivation therapy in prostate cancer and metabolic risk for atherosclerosis. J Clin Endocrinol Metab 2008; 93 : 2042-9.

5 Chodak GW, Keane T, Klotz L. Critical evaluation of hormonal therapy for carcinoma of the prostate. Urology 2002; 60: 201-8.

6 Moul JW, Bañez LL, Freedland SJ. Rising PSA in nonmetastatic prostate cancer. Oncology (Williston Park) 2007; 21 : 1436-45.

7 Shahinian VB, Kuo YF, Freeman JL, Orihuela E, Goodwin JS. Increasing use of gonadotropin-releasing hormone agonists for the treatment of localized prostate carcinoma. Cancer 2005; 103: 1615-24.

8 Basaria S, Lieb J 2nd, Tang AM, DeWeese T, Carducci M et al. Long term effects of androgen deprivation therapy in prostate cancer patients. Clin Endocrinol 2002; 56: 779-86.

9 Smith MR, Finkelstein JS, McGovern FJ, Zietman AL, Fallon MA et al. Changes in body composition during androgen deprivation therapy for prostate cancer. J Clin Endocrinol Metab 2002; 87: 599-603.

10 Bhasin S, Taylor WE, Singh R, Artaza J, Sinha-Hikim I et al. The mechanisms of androgen effects on body composition: mesenchymal pluripotent cell as the target of androgen action. J Gerontol A Biol Sci Med Sci2003; 58: M1103-10.

11 Smith JC, Bennett S, Evans M, Kynaston HG, Parmar M et al. The effects of induced hypogonadism on arterial stiffness, body composition, and metabolic parameters in males with prostate cancer. J Clin Endocrinol Metab 2001; 86: 4261-7.

12 Van London GJ, Levy ME, Perera S, Nelson JB, Greenspan SL. Body composition changes during androgen deprivation therapy for prostate cancer: a 2-year prospective study. Crit Rev Oncol Hematol 2008; 68: 172-7.

13 Harle LK, Maggio M, Shahani S, Braga-Basaria M, Basaria S. Endocrine Complications of androgen deprivation therapy in men with prostate cancer. Clin Adv Hematol Oncol 2006; 4: 687-96

14 Smith MR, Lee H, Nathan DM. Insulin sensitivity during combined androgen blockade for prostate cancer. J Clin Endocrinol Metab 2006; 91: 1305-8.

15 Nishiyama T, Ishizaki F, Tsutomu A, Shimura H, Takahashi K. The influence of androgen deprivation therapy on metabolism in patients with prostate cancer. J Clin Endocrinol Metab 2005; 90: 657-60.

16 Basaria S, Muller DC, Carducci MA, Egan J, Dobs AS. Hyperglycemia and insulin resistance in men with prostate carcinoma who receive androgen deprivation therapy. Cancer 2006; 106: 581-8.

17 Basaria S, Muller DC, Carducci MA, Egan J, Dobs AS. Relation between duration of androgen deprivation therapy and degree of insulin resistance in men with prostate cancer. Arch Intern Med 2007; 167: 612-3.

18 Keating NL, O'Malley AJ, Smith MR. Diabetes and cardiovascular disease during androgen deprivation therapy for prostate cancer. J Clin Oncol 2006; 24: 4448-56. 
19 Alibhai SM, Duong-Hua M, Sutradhar R, Fleshner NE, Warde P et al. Impact of androgen deprivation therapy on cardiovascular disease and diabetes. J Clin Oncol 2009; 27: 3452-8.

20 Keating N, O'Malley AJ, Freedland SJ, Smith MR. Diabetes and cardiovascular disease with androgen deprivation therapy: observational study of veterans with prostate cancer. J Nat/ Cancer Inst 2010; 102: 39-46.

21 Alberti KG, Eckel RH, Grundy SM, Zimmet PZ, Cleeman JI et al. Harmonizing the metabolic syndrome: a joint interim statement of the International Diabetes Federation Task Force on Epidemiology and Prevention; National Heart, Lung, an Blood Institute; American Heart Association; World Heart Federation; Internationa Atherosclerosis Society; and International Association for the Study of Obesity. Circulation 2009; 120: 1640-5.

22 Lakka HM, Laaksonen DE, Lakka TA, Niskanen LK, Kumpusalo E et al. The metabolic syndrome and total and cardiovascular disease mortality in middle aged men. JAMA 2002; 288: 2709-16.

23 Laaksonen DE, Niskanen L, Punnonen K, Nyyssönen K, Tuomainen TP et al. Testosterone and sex hormone-binding globulin predict the metabolic syndrome and diabetes in middle aged men. Diabetes Care 2004; 27: 1036-41.

24 Braga-Basaria M, Dobs AS, Muller DC, Carducci MA, John M et al. Metabolic syndrome in men with prostate cancer undergoing long-term androgen deprivation therapy. J Clinl Oncol 2006; 24: 3979-83.

25 Araujo AB, Dixon JM, Suarez EA, Murad MH, Guey LT et al. Endogenous testosterone and mortality in men: a systematic review and meta-analysis. J Clin Endocrinol Metab 2011; 96: 3007-19.

26 Phillips GB, Pinkernell BH, Jing TY. The association of hypotestosteronemia with coronary artery disease in men. Arterioscler Thromb 1994; 14: 701-6.

27 Garnick MB, Pratt CM, Campion M, Shipley J. The effect of hormonal therapy for prostate cancer on electrocardiographic QT interval: phase 3 results following treatment with leuprolide and goserelin, alone or with bicalutamide, and the GnRG antagonist abarelix. J Clin Oncol 2004; 22: 4578.

28 Smith MR, Klotz L, Persson BE, Olesen TK, Wilde AA. Cardiovascular safety of degarelix: results from a 12-month, comparative, randomized, open label, paralle group phase III trial in patients with prostate cancer. J Uro/ 2010; 184: 2313-9.

29 Tsai HK, D'Amico AV, Sadetsky N, Chen MH, Carroll PR. Androgen deprivation therapy for localized prostate cancer and the risk of cardiovascular mortality. J Nat/ Cancer Inst 2007; 99: 1516-24.
30 Saigal CS, Gore JL, Krupski TL, Hanley J, Schonlau M et al. Androgen deprivation therapy increases cardiovascular morbidity in men with prostate cancer. Cancer2007, 110: $1493-500$

31 Nanda A, Chen MH, Braccioforte MH, Moran BJ, D’Amico AV. Hormonal therapy use for prostate cancer and mortality in men with coronary artery disease-induced congestive heart failure or myocardial infarction. JAMA 2009; 302: 866-73.

32 Azoulay L, Yin H, Benayoun S, Renoux C, Boivin JF et al. Androgen deprivation therapy and the risk of stroke in patients with prostate cancer. Eur Urol 2011;60 1244-50.

33 Ehdaie B, Atoria CL, Gupta A, Feifer A, Lowrance WT et al. Androgen deprivation and thromboembolic events in men with prostate cancer. Cancer; e-pub ahead of print 9 November 2011; doi: 10.1002/cncr.26623.

34 Efstathiou JA, Bae K, Shipley WU, Hanks GE, Pilepich MV et al. Cardiovascular mortality and duration of androgen deprivation for locally advanced prostate cancer: analysis of RTOG 92-02. Eur Urol 2008; 54: 816-23.

35 Kim J, Vaid M, Tyldesley S, Woods R, Pickles T. Population-based study of cardiovascular mortality among patients with prostate cancer treated with radical external bean radiation therapy with and without adjuvant androgen deprivation therapy at the Birtish Columbia Cancer Agency. Int J Radiat Oncol Biol Phys 2011 80: 742-50.

36 Punnen S, Cooperberg MR, Sadetsky N, Carroll PR. Androgen deprivation therapy and cardiovascular risk. J Clin Oncol 2011; 29: 3510-6.

37 Chung AD, Chen YK, Wu FJ, Lin HC. Hormone therapy for prostate cancer and the risk of stroke: a 5-year follow-up study. BJU Int; e-pub ahead of print 24 August 2011; doi: 10.1111/j.1464-410X.2011.10459.x.

38 Levine GN, D'Amico AV, Berger P, Clark PE, Eckel RH et al. Androgen-deprivation therapy in prostate cancer and cardiovascular risk: a science advisory from the American Heart Association, American Cancer Society, and American Urological Association: endorsed by the American Society for Radiation Oncology. CA Cancer J Clin 2010; 60: 194-201.

39 Nobes JP, Langley SE, Klopper T, Russell-Jones D, Laing RW. A prospective randomized pilot study evaluating effects of metformin and lifestyle interventions on patients with prostate cancer receiving androgen deprivation therapy. BJU Int e-pub ahead of print 20 September 2011; doi: 10.1111/j.1464-410X.2011. $10555 x$ 